\title{
Photoautotrophic production of D-lactic acid in an engineered cyanobacterium
}

Arul M Varman ${ }^{2^{*}+}, Y i u^{1,2 \dagger}$, Le You ${ }^{2}$ and Yinjie J Tang ${ }^{2^{*}}$

\begin{abstract}
Background: The world faces the challenge to develop sustainable technologies to replace thousands of products that have been generated from fossil fuels. Microbial cell factories serve as promising alternatives for the production of diverse commodity chemicals and biofuels from renewable resources. For example, polylactic acid (PLA) with its biodegradable properties is a sustainable, environmentally friendly alternative to polyethylene. At present, PLA microbial production is mainly dependent on food crops such as corn and sugarcane. Moreover, optically pure isomers of lactic acid are required for the production of PLA, where D-lactic acid controls the thermochemical and physical properties of PLA. Henceforth, production of D-lactic acid through a more sustainable source $\left(\mathrm{CO}_{2}\right)$ is desirable.

Results: We have performed metabolic engineering on Synechocystis sp. PCC 6803 for the phototrophic synthesis of optically pure D-lactic acid from $\mathrm{CO}_{2}$. Synthesis of optically pure D-lactic acid was achieved by utilizing a recently discovered enzyme (i.e., a mutated glycerol dehydrogenase, $\mathrm{GlyDH}^{*}$ ). Significant improvements in D-lactic acid synthesis were achieved through codon optimization and by balancing the cofactor (NADH) availability through the heterologous expression of a soluble transhydrogenase. We have also discovered that addition of acetate to the cultures improved lactic acid production. More interestingly, ${ }^{13} \mathrm{C}$-pathway analysis revealed that acetate was not used for the synthesis of lactic acid, but was mainly used for synthesis of certain biomass building blocks (such as leucine and glutamate). Finally, the optimal strain was able to accumulate $1.14 \mathrm{~g} / \mathrm{L}$ (photoautotrophic condition) and $2.17 \mathrm{~g} / \mathrm{L}$ (phototrophic condition with acetate) of D-lactate in 24 days.

Conclusions: We have demonstrated the photoautotrophic production of D-lactic acid by engineering a cyanobacterium Synechocystis 6803. The engineered strain shows an excellent D-lactic acid productivity from $\mathrm{CO}_{2}$. In the late growth phase, the lactate production rate by the engineered strain reached a maximum of $\sim 0.19 \mathrm{~g}$ D-lactate/L/day (in the presence of acetate). This study serves as a good complement to the recent metabolic engineering work done on Synechocystis 6803 for L-lactate production. Thereby, our study may facilitate future developments in the use of cyanobacterial cell factories for the commercial production of high quality PLA.
\end{abstract}

\section{Background}

Fossil fuels helped literally ignite the industrial revolution, and from then on radically changed the way we live; today, thousands of products are generated from fossil fuels [1]. Unfortunately, fossil fuels are nonrenewable and their reserves will foreseeably run dry. Moreover, the reckless use of this resource has resulted in a tremendous release of greenhouse gases leading to adverse effects to our earth's climate and to the creatures

\footnotetext{
* Correspondence: avarman@go.wustl.edu; yinjie.tang@seas.wustl.edu ${ }^{\dagger}$ Equal contributors

${ }^{2}$ Department of Energy, Environmental and Chemical Engineering, Washington University, St. Louis, MO 63130, USA

Full list of author information is available at the end of the article
}

living on our planet. These drawbacks have driven researchers to look for alternative renewable replacements for petroleum and petroleum-derived products. Amongst the petroleum-derived products; polyethylene with an annual productivity of 80 million metric tons per annum stands out as one of the most commonly used plastics [2]. Polylactic acid (PLA) is made by the polymerization of lactic acid and has the potential to replace polyethylene as a biodegradable alternative [3]. Lactic acid is a chiral compound and exists in two isomeric forms: $\mathrm{D}(-)$ lactic acid and $\mathrm{L}(+)$ lactic acid. The various properties of polylactic acid are modulated by the mixing ratio of the $\mathrm{D}(-)$ and L (+) lactic acid and, henceforth, it is essential to produce both the isomers [4]. It has been estimated that for the 
PLA production to be profitable, the lactic acid price should be less than $0.8 \$ / \mathrm{kg}$ [5]. This necessitates the production of lactic acid from a cheaper source. Although microbial fermentation can produce lactate from sugarbased feedstock, such process may compete with global food supplies. Therefore, this work focuses on cyanobacterial process development for the sustainable synthesis of D (-) lactic acid, with $\mathrm{CO}_{2}$ as the carbon substrate and sunlight as an energy source.

Cyanobacteria have the ability to reduce atmospheric $\mathrm{CO}_{2}$ into useful organic compounds by using solar energy and have been engineered to synthesize a number of valueadded products [6-9]. Synechocystis sp. PCC 6803 (hereafter Synechocystis 6803) with its ability to uptake foreign DNA naturally, has been the model organism of choice for various metabolic engineering works [10-12]. Synechocystis 6803 also has the ability to grow mixotrophically with glucose and acetate [13]. Therefore, along with $\mathrm{CO}_{2}$, its versatile carbon metabolism allows the co-utilization of cheap organic compounds for product biosynthesis. For example, acetate abundant wastewater generated from biomass hydrolysis and anaerobic digestion [14] can be potentially used for promoting cyanobacterial productivity. More importantly, there are numerous molecular biology tools for Synechocystis 6803, making it an attractive organism for metabolic engineering works $[15,16]$.

Synechocystis 6803 has recently been engineered for the production of L-lactate (a maximal titer of $1.8 \mathrm{~g} / \mathrm{L}$ and a maximal productivity of $0.15 \mathrm{~g} / \mathrm{L} /$ day) [17-19]. However, engineering Synechocystis 6803 for the production of optically pure D-lactate synthesis is more difficult due to the lack of an efficient D-lactate dehydrogenase. Recently, a mutated glycerol dehydrogenase (GlyDH*) was discovered by Wang et al. [20] and this enzyme was found to behave as a D-lactate dehydrogenase, exhibiting an unusually high specific activity of 6.9 units per mg protein with pyruvate and $\mathrm{NADH}$ as substrates. This enzyme allows a Bacillus coagulans strain to produce $90 \mathrm{~g} / \mathrm{L}$ of D-lactate. Their work served as a motivation for us to engineer Synechocystis 6803 through the heterologous expression of gldA101 (encodes GlyDH*). We found that this original enzyme was able to synthesize optically pure D-lactate in Synechocystis 6803. To further improve cyanobacterial productivity, we employed three strategies: 1. Codon optimization of gldA101 (Additional file 1: Figure S1); 2. Heterologous expression of a transhydrogenase; 3. Supplementing cultures with extracellular carbon sources (such as glucose, pyruvate and acetate). The final engineered strain demonstrated a high D-lactic acid productivity and titer (titer $>1 \mathrm{~g} / \mathrm{L}$ ).

\section{Results and discussion}

Cyanobacteria need a lactate dehydrogenase to synthesize lactate from pyruvate (Figure 1). Earlier works on Synechocystis
6803 for lactate production involved the expression of an ldh from Bacillus subtilis for synthesis of L-lactate [18]. As a first step, we tested the activity of GlyDH* for D-lactate production [20] by transferring the gene from Bacillus coagulans to Synechocystis 6803. A plasmid pYY1 was constructed that contained the gene gldA101 under the control of an Isopropyl $\beta$-D-1-thiogalactopyranoside (IPTG) inducible promoter, $\mathrm{P}_{\text {trc }}$. The gldA101 gene was then subsequently transferred to the glucose tolerant wild type Synechocystis 6803 through natural transformation, generating the strain AV08. The optical density and the D-lactate concentration of the AV08 cultures were monitored in shake flasks. As can be verified from Figure 2, AV08 did not show any significant levels of D-lactate in the initial 12 days. The D-lactate levels started increasing steadily at the late autotrophic growth phase and reached a final titer of $0.4 \mathrm{~g} / \mathrm{L}$, whereas a wild type strain of Synechococcus 7002 was able to produce only $\sim 7 \mathrm{mg} / \mathrm{L}$ of D-lactate through glucose fermentation [21].

A familiar strategy to increase the synthesis of a target product would be to increase the levels of the heterologous enzyme inside the cell. This can be achieved by modifying the enzyme regulation either at the transcriptional level or at the translational level. Cyanobacteria are known to have their own preference in the use of codons for synthesizing amino acids [24]. Lindberg et al. [25] have employed codon optimization for the isoprene synthase gene IspS and have found a 10-fold increase in the IspS expression level. More recently, this strategy was applied to increase the expression of the efe gene (from Pseudomonas syringae) in Synechocystis 6803 for ethylene production [26]. Since the gene involved in this work was borrowed from a gram-positive organism and Synechocystis 6803 being gram-negative, we hypothesized that this would be a useful strategy. The codon optimized gene gldA101-syn (synthesized by Genewiz Inc, South Plainfield, NJ) was integrated into the psbA1 gene loci in the genome of the WT Synechocystis 6803 using the plasmid pDY3 to obtain the strain AV11.

Further improvements in product synthesis can be achieved by rectification of bottlenecks in the metabolic pathway. The lactate dehydrogenase enzyme utilizes $\mathrm{NADH}$ as its cofactor, whereas the ratio of NADH to NADPH is reported to be much lower in cyanobacteria. For example, the ratio of NADH to NADPH in Synechococcus 7942 under light conditions was estimated to be 0.15, and in Synechocystis 6803 under photoautotrophic conditions the intracellular NADH concentration was only $20 \mathrm{nmol} / \mathrm{g}$ fresh weight, whereas the intracellular $\mathrm{NADPH}$ concentration was about $140 \mathrm{nmol} / \mathrm{g}$ fresh weight [27-29]. This lower concentration of $\mathrm{NADH}$ in cyanobacteria, points to the fact that availability of NADH could be a major limiting factor for synthesizing 


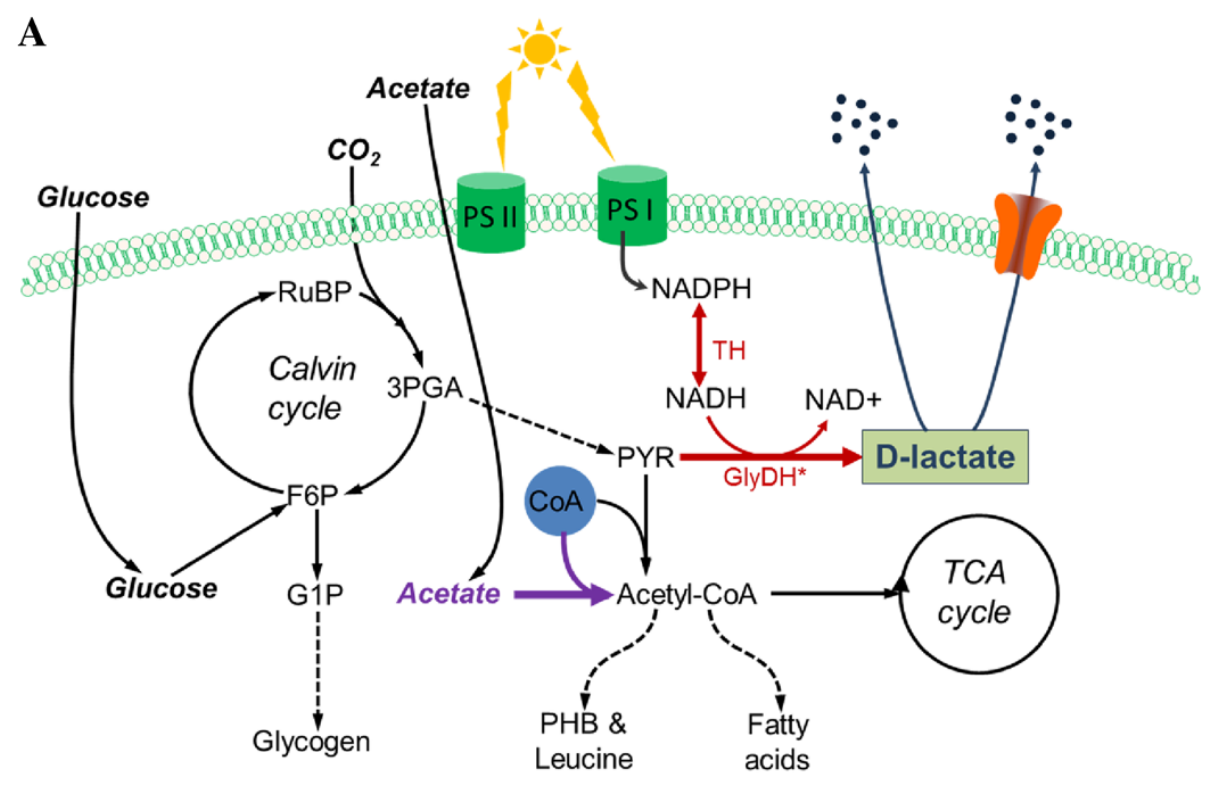

\section{B}

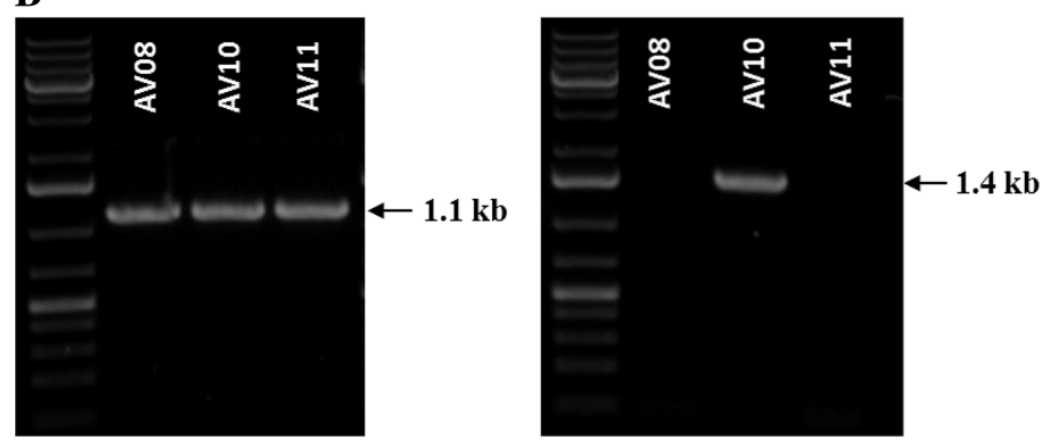

Figure 1 Metabolic engineering of Synechocystis $\mathbf{6 8 0 3}$ for the synthesis of D-lactic acid. (A) Metabolic pathway for D-lactate synthesis. Lactate permeation through the cell membrane occurs either via a lactate transporter or by passive diffusion [22,23]. Red arrows indicate the heterologous pathway engineered into Synechocystis 6803. Abbreviations: GlyDH*, mutant glycerol dehydrogenase; TH, Transhydrogenase; 3PGA,

3-phosphoglycerate; CoA, Coenzyme A; G1P, glucose 1-phosphate; F6P, fructose 6-phosphate; PHB, poly- $\beta$-hydroxybutyrate; RuBP, ribulose 1, 5-bisphosphate. (B) Colony PCR to verify the presence of the heterologous genes of the mutant glycerol dehydrogenase (Left picture) and transhydrogenase (Right picture) in the engineered strains of Synechocystis 6803. gldA101 was amplified with primers gldA-o-F3 and gldA-o-R; gldA101-syn was amplified with primers gldA-o-F and gldA-o-R2; sth was amplified with primers tranNADH-F and tranNADH-R (Table 1).

D-lactate. Henceforth, a soluble transhydrogenase, sth from Pseudomonas aeruginosa [30], was introduced downstream of the gene gldA101-syn. This engineered strain was called AV10. The heterologous genes in AV10 and AV11 are under the control of the same single promoter, $\mathrm{P}_{\text {trc }}$, located upstream of gldA101-syn and sth in AV10 and located upstream of gldA101-syn in AV11.

The three strains (AV08, AV10 and AV11) showed similar growth rates to wild type strain under photoautotrophic conditions, and thus the production of D-lactate did not introduce growth defects in the engineered strains (Figure 2A and Additional file 1: Figure S2). However, the three strains differed in the production rate of D-lactic acid. The strain AV11 with codon optimization (gldA101syn) had an improved productivity for D-lactate compared to the AV08 strain (Figure 2B). Both strains produced D-lactate mainly during the later growth stage. Introduction of the transhydrogenase improved the D-lactate synthesis further in AV10, and this strain produced D-lactate in both the growth phase and non-growth phase. The rate of photoautotrophic D-lactate production by AV10 increased significantly (achieving a maximum productivity of $\sim 0.1 \mathrm{~g} / \mathrm{L} /$ day and $\sim 0.2 \mathrm{mmol} / \mathrm{g}$ cell/day) during the late phase of the culture and the final titer of D-lactate reached $1.14 \mathrm{~g} / \mathrm{L}$.

We observed that the D-lactate production rate reached its peak in the later stages of cultivation, suggesting that more carbon flux has been directed to lactate production during the non-growth phase. This increased flux was expected because the lactate precursor (pyruvate) is a key 


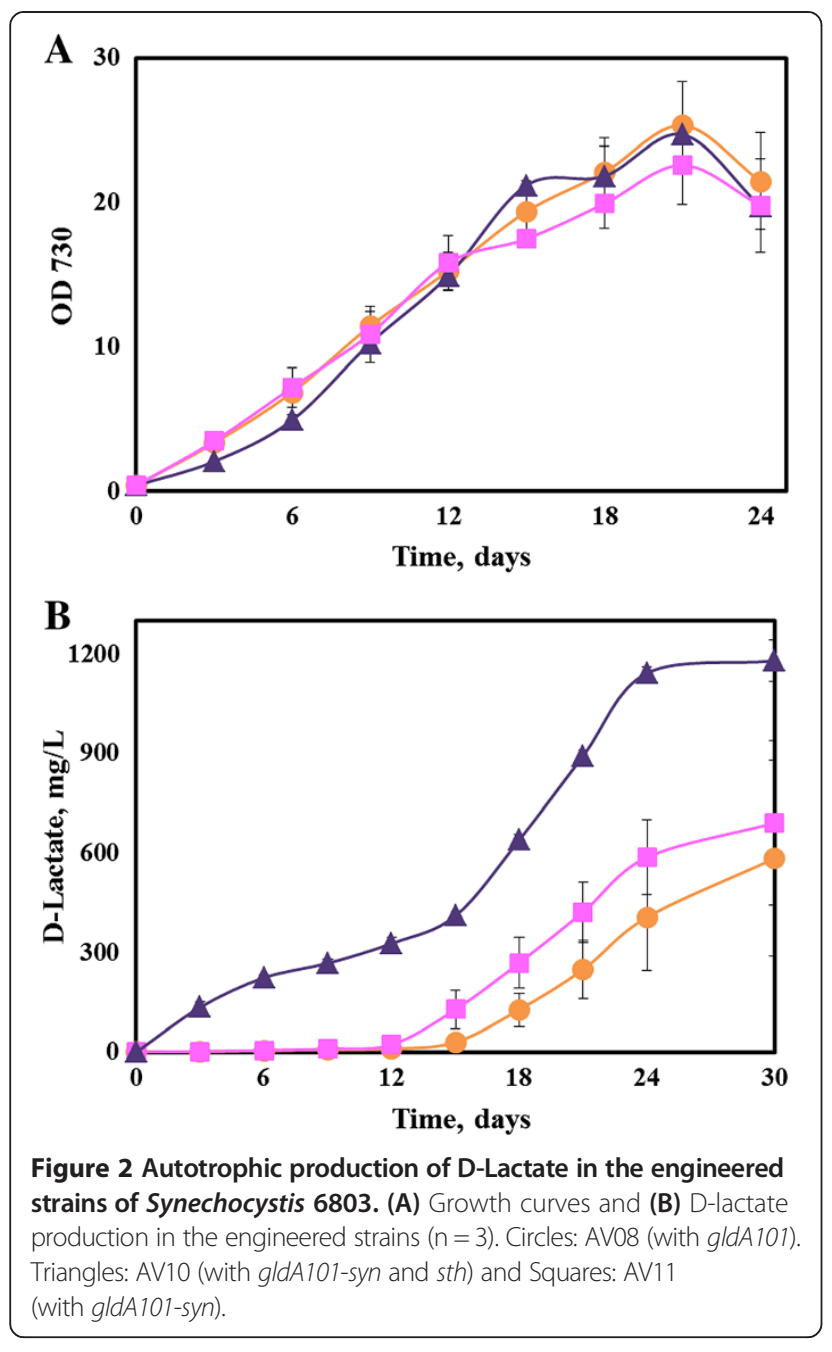

metabolic node occupying a central position in the synthesis of diverse biomass components, and more pyruvate becomes available for lactate synthesis when biomass growth becomes slow. Therefore, an obvious thought would be to enhance lactate production by supplementing the cultures with pyruvate [31]. However, our experiments found that addition of pyruvate did not yield apparent improvements in D-lactate synthesis (data not shown), possibly because Synechocystis 6803 may lack an effective pyruvate transporter. The alternate option would be to grow AV10 with glucose and increase the glycolysis flux for pyruvate synthesis. In our previous study, addition of glucose was found to increase isobutanol production in Synechocystis 6803 [32]. However in this study, when we grew the AV10 strain under mixotrophic conditions (with $5 \mathrm{~g} / \mathrm{L}$ glucose), it did not show a higher growth rate or display improvements in the final D-lactate titer compared to the autotrophic condition. The AV10 cultures grown in the presence of glucose instead showed an impaired growth, possibly because the engineered pathways caused a metabolic imbalance during glucose catabolism (Figure 3).
We also hypothesized that the intracellular pyruvate pool can be increased for lactate production by addition of exogenous acetate. Supplementing cultures with acetate can redirect more carbon from pyruvate to lactate in three possible ways [33]: (1) acetate is used as a building block for lactate production; (2) acetate provides additional carbon source for biomass synthesis and reduce pyruvate consumption; (3) acetate conversion by acetyl-CoA synthetase consumes Coenzyme-A (CoA), decreasing the CoA pool available for pyruvate decarboxylation. To test this hypothesis, the AV10 cultures were supplemented with $15 \mathrm{mM}$ acetate. We found that growth rate of the AV10 cultures with acetate (Figure 3A) remained comparable to their growth rate under autotrophic condition, but there was substantial improvement in the synthesis of D-lactate (the maximal titer reached $2.17 \mathrm{~g} / \mathrm{L}$ and the peak productivity reached $\sim 0.19 \mathrm{~g} / \mathrm{L} /$ day, Figure $3 \mathrm{~B}$ ).

To further understand the role played by glucose and acetate in D-lactate synthesis, AV10 cultures were grown with $\left[1,2-{ }^{13} \mathrm{C}\right]$ glucose and $\left[1,2-{ }^{13} \mathrm{C}\right]$ acetate (Sigma, St. Louis). Cultures were collected from the mid-log phase and were used for amino acid and D-lactate analysis. As
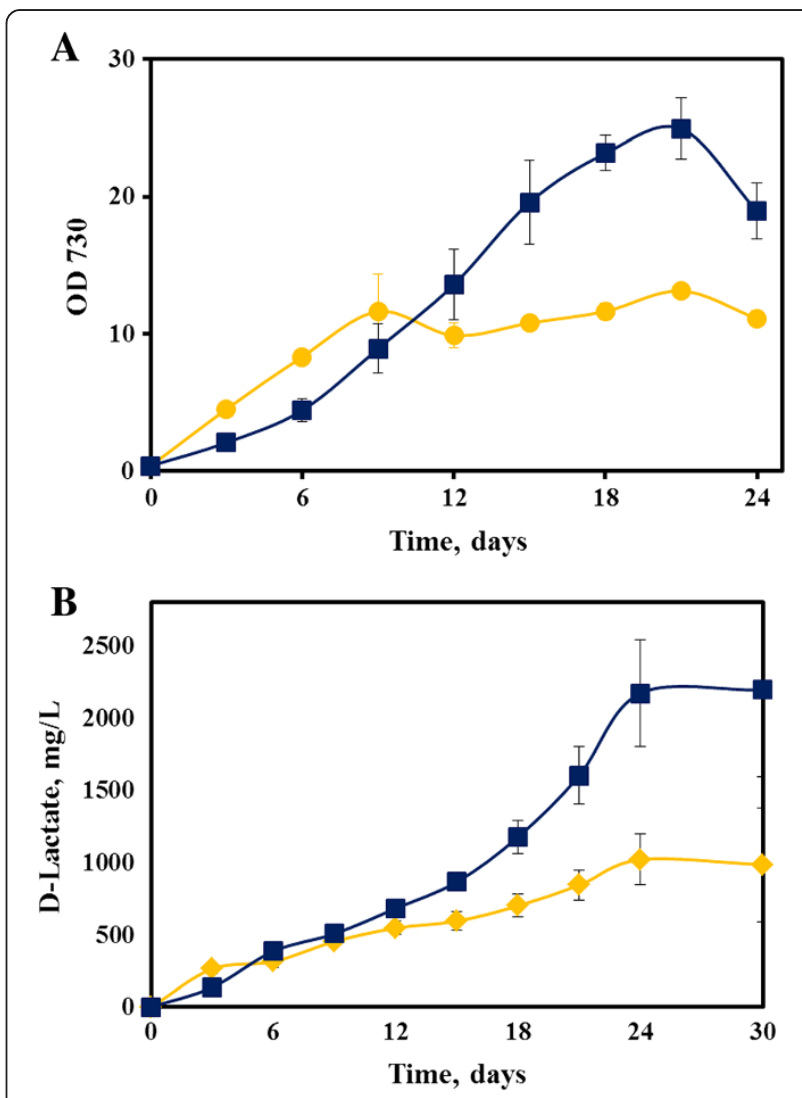

Figure 3 Mixotrophic production of D-Lactate by AV10. (A)

Growth and (B) D-lactate production in the engineered Synechocystis 6803 strain AV10 $(n=3)$, with the provision of additional organic carbon source, i.e., with glucose and acetate (Mixotrophic metabolism). Squares: with acetate. Circles: with glucose. 
an example, mass spectrum of D-lactate from a cyanobacterial culture is shown in Additional file 1: Figure S3. The ${ }^{13} \mathrm{C}$ abundance in the amino acids and lactate were obtained as mass fraction $\mathrm{m}_{\mathrm{i}}$, where ' $\mathrm{i}$ ' indicates the number of ${ }^{13} \mathrm{C}$ in the molecule. As can be seen from Figure 4A, glucose-fed cells have significant ${ }^{13} \mathrm{C}$-carbon distributed in amino acids (indicated by an increase in $\mathrm{m}_{1}$ and $\mathrm{m}_{2}$ ). Also, D-lactate from glucose-fed cultures was partially ${ }^{13} \mathrm{C}$-labeled $\left(\mathrm{m}_{2} \sim 0.22\right)$. The isotopomer data in Figure $4 \mathrm{~A}$ proved that ${ }^{13} \mathrm{C}$-glucose provided the carbon source for both biomass and lactate production. However, glucose-based mixotrophic fermentation is not beneficial to D-lactate production compared to autotrophic cultures, possibly because carbon flux from glycolysis may cause some carbon and energy imbalance [32]. As for the acetate-fed cultures, only leucine and glutamate (which both use acetyl-CoA as their precursor) were significantly labeled (an $\mathrm{m}_{2}$ of 0.31 and 0.32 respectively), while other amino acids (e.g., aspartate and alanine) were nonlabeled
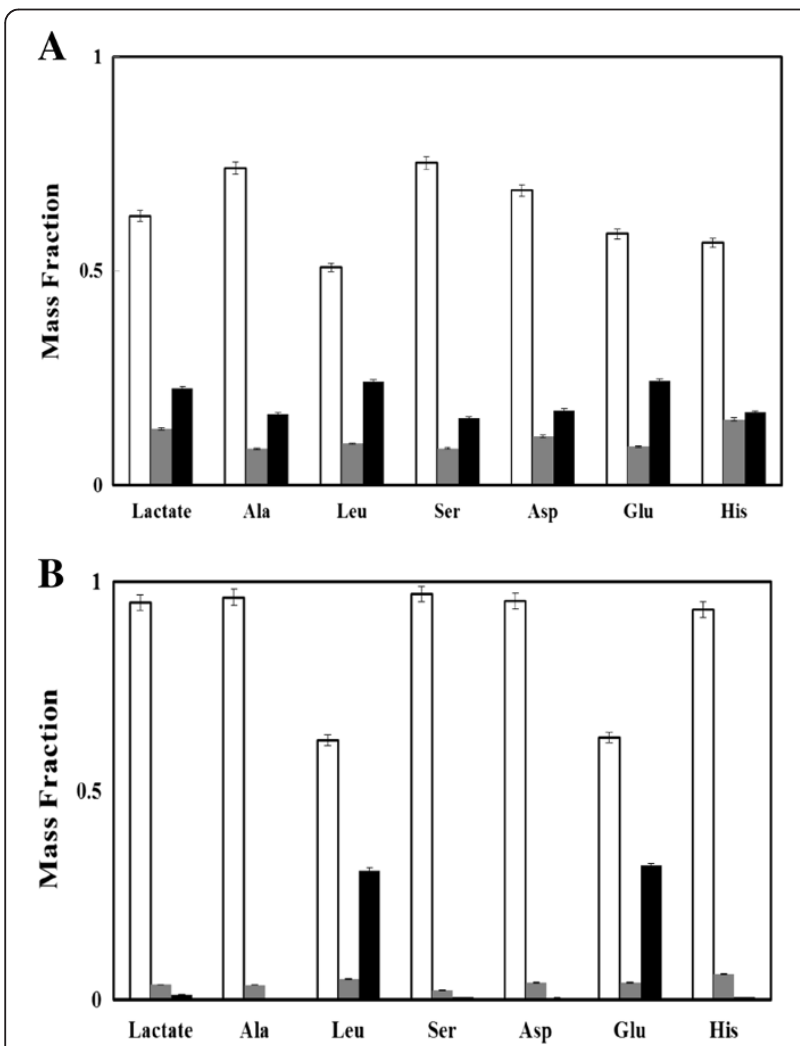

Figure 4 Isotopomer analysis showing the mass fraction of isotopomers for selected proteinogenic amino acids [TBDMS based measurement] and D-lactate [MSTFA based measurement]. Standard abbreviations are used for amino acids in the figure. (A) Cultures grown with $5 \mathrm{~g} / \mathrm{L}$ of $\left[1,2-{ }^{13} \mathrm{C}\right]$ glucose and (B) Cultures grown with $15 \mathrm{mM}$ of $\left[1,2-{ }^{13} \mathrm{C}\right]$ acetate. "white bar" $\mathrm{m}_{0}$ mass fraction without any labeled carbon; "grey bar" $m_{1}$ - mass fraction with one labeled carbon; "black bar" $m_{2}$ - mass fraction with two labeled carbon. (Note: natural ${ }^{13} \mathrm{C}$ makes up about $1.1 \%$ of total carbon as measurement background).
(Figure 4B). Interestingly, D-lactate from acetate-fed culture was almost nonlabeled, indicating that the carbons of lactate molecules were mainly derived from $\mathrm{CO}_{2}$. Therefore, the observed enhancement of lactate synthesis in the presence of acetate can be explained by two complementary mechanisms. First, acetate is an additional carbon source for synthesizing biomass building blocks, such as fatty acids and some amino acids, thus redirecting the extra carbon flux from $\mathrm{CO}_{2}$ to lactate. Secondly, acetate may limit the pyruvate decarboxylation reaction by reducing the CoA pool by the formation of acetyl-CoA and thus improve pyruvate availability for lactate synthesis.

\section{Conclusions}

The results reported here are for the autotrophic production of D-lactate in cyanobacteria via the heterologous expression of a novel D-lactate dehydrogenase $\left(\mathrm{GlyDH}^{*}\right)$ and by balancing the precursors and cofactors. Other molecular strategies may also be applied to further improve the D-lactate production: (1) by seeking stronger promoters [16]; (2) optimizing ribosomal binding sites [34]; (3) improving activity of $\mathrm{GlyDH}^{*}$ via protein engineering; (4) introducing powerful lactate transporter [22]; (5) knocking out competing pathways (such as the glycogen and polyhydroxybutyrate synthesizing pathways); (6) duplicating the heterologous genes by integrating at multiple sites [35]; and (7) limiting biomass production by knocking down the pyruvate decarboxylation reaction. Also, considering the future outdoor algal processes for scaled up D-lactate production, we hypothesize that knocking out metabolic pathways that synthesize carbon storage molecules (polyhydroxybutyrate and glycogen) may be deleterious to algal growth during the night phase in day-night cultivation [36]. On the other hand, process optimization by employing better light conditions, along with proper $\mathrm{CO}_{2}$ concentration, $\mathrm{pH}$ and temperature control, may also be employed to increase the D-lactate productivity in a scaled-up system.

\section{Materials and methods}

\section{Chemicals and reagents}

Restriction enzymes, Phusion DNA polymerase, T4 DNA ligase and 10-Beta electro-competent E. coli kit were purchased from Fermentas or New England BioLabs. Oligonucleotides were purchased from Integrated DNA Technologies (IDT). All organic solvents, chemicals, ${ }^{13} \mathrm{C}$ labeled acetate, and glucose used in this study were purchased from Sigma-Aldrich (St. Louis, MO).

\section{Medium and growth conditions}

E. coli strain 10-Beta was used as the host for all plasmids constructed in this study. E. coli cells were grown in liquid Luria-Bertani (LB) medium at $37^{\circ} \mathrm{C}$ in a shaker at $200 \mathrm{rpm}$ or on solidified LB plates. Ampicillin 
Table 1 Primer sequences

\begin{tabular}{|c|c|}
\hline Primer name & Sequence $\left(5^{\prime} \rightarrow 3^{\prime}\right)$ \\
\hline gldA-o-F & GGATCCTTGACAATTAATCATCCGGCTCG \\
\hline gldA-o-F2 & $\begin{array}{l}\text { GGATCCTTGACAATTAATCATCCGGCTCGTATAATGTGTGGA } \\
\text { ATTGTGAGCGGATAACAATTCACACAGGAGATATAATCAT } \\
\text { ATGACGAAAATCATTACCTCTCCAAGCAAGTTATACAAGG }\end{array}$ \\
\hline gldA-o-F3 & ATGACGAAAATCATTACCTCTCCAAG \\
\hline gldA-o-R & GCTAGCTCATGCCCATITITCCTTATAATACCGCCCG \\
\hline gldA-o-R2 & TTAGGCCCACTTTCCTTGTAATAGC \\
\hline $\operatorname{tranNADH-F}$ & CCTAAGCTAGCGGAGGACTAGCATGG \\
\hline $\operatorname{tranNADH-R}$ & GCTAGCGGTACCTCAAAAAAGCCGG \\
\hline ptka3-F & CCCGAAGTGGCGAGCCCGAT \\
\hline $\mathrm{CO}-\mathrm{F}$ & TTGATGTTGCCTTTGAACCC \\
\hline O-F & ATGGATACGAAAGTGATTGC \\
\hline sth-F & GAGCTACCACCTGCGCAACA \\
\hline AMV17R & GCGCGACTCCCCGTCTITGACTATCCTIITAGGATGGGGCA \\
\hline ps1_up_fwda & TACCGGAACAGGACCAAGCCTT \\
\hline
\end{tabular}

$(100 \mu \mathrm{g} / \mathrm{mL})$ or kanamycin $(50 \mu \mathrm{g} / \mathrm{mL})$ was added to the LB medium when required for propagation of the plasmids in E. coli. The wild-type (glucose-tolerant) and the recombinant strain of Synechocystis 6803 were grown at $30^{\circ} \mathrm{C}$ in a liquid blue-green medium (BG-11 medium) or on solid BG-11 plates at a light intensity of $100 \mu \mathrm{mol}$ of photons $\mathrm{m}^{-2} \mathrm{~s}^{-1}$ in ambient air. Kanamycin $(20 \mu \mathrm{g} / \mathrm{mL})$ was added to the BG-11 growth medium as required. Growth of the cells was monitored by measuring their optical density at $730 \mathrm{~nm}\left(\mathrm{OD}_{730}\right)$ with an Agilent Cary $60 \mathrm{UV}$-vis spectrophotometer. $10 \mathrm{~mL}$ cultures for the synthesis of D-lactate were grown (initial $\mathrm{OD}_{730}, 0.4$ ) in $50-\mathrm{mL}$ shake flasks without any antibiotic and $1 \mathrm{mM}$
Isopropyl $\quad \beta$-D-1-thiogalactopyranoside (IPTG) was added for induction. Mixotrophic cultures of Synechocystis 6803 were started in BG-11 medium containing a known amount of glucose $(0.5 \%)$ or acetate $(15 \mathrm{mM})$ as an organic carbon source.

\section{Plasmid construction and transformation}

The vector pTKA3 [32] served as the backbone for all the plasmids constructed in this study. The gene gldA101 encoding GlyDH* [20], was amplified from the plasmid pQZ115 with the primers gldA-o-F2 and gldA$\mathrm{o}-\mathrm{R}$ (Tables 1 and 2). The obtained $1.2 \mathrm{~kb}$ fragment was digested with BamHI/NheI and cloned into the same restriction sites of pTKA3, yielding the vector pYY1. A gene cassette, which consists of the codon optimized gldA101 (i.e., gldA101-syn) with the promoter $\mathrm{P}_{\text {trc }}$ in the upstream and the transhydrogenase (sth) gene from Pseudomonas aeruginosa [30] in the downstream, was chemically synthesized by Genewiz Inc (South Plainfield, NJ) and cloned into the commonly used E. coli vector pUC57-kan resulting in the plasmid vector pUC57glda_sth. The vector pUC57-glda_sth was digested with BamHI/NheI, and the yielding $2.6 \mathrm{~kb}$ fragment was cloned into the corresponding restriction sites of pTKA3, resulting in the vector $\mathrm{pDY} 2$. The vector $\mathrm{pDY} 3$ was constructed by self-ligation of the $8.2 \mathrm{~kb}$ fragment obtained through the digestion of pDY2 with KpnI.

Natural transformation of Synechocystis 6803 was performed by using a double homologous-recombination procedure as described previously [38]. Recombinant colonies appeared between 7 and 10 days post inoculation. The genes of interest were finally integrated into the psbA1 gene loci (a known neutral site under normal growth conditions) in the genome of Synechocystis 6803

Table 2 Plasmids and strains

\begin{tabular}{|c|c|c|}
\hline Plasmids/Strains & Description & Source or reference \\
\hline \multicolumn{3}{|c|}{ Plasmids } \\
\hline pUC57-glda_sth & Chemically synthesized gene cassette consisting of $P_{\text {tra }}$ gldA101-syn and sth. & Genewiz; [20,30,37] \\
\hline pQZ115 & Plasmid carrying gldA101 & {$[20]$} \\
\hline PTKA3 & Backbone plasmid for all vectors constructed in this study, with psbA1 as the integration loci. & {$[32]$} \\
\hline pYY1 & Derived from pTKA3 with gldA101 and the promoter, $\mathrm{P}_{\text {trc. }}$ & This study \\
\hline pDY2 & Derived from pTKA3 with gldA101-syn, sth and the promoter, $\mathrm{P}_{\text {trc }}$. & This study \\
\hline pDY3 & Derived from pTKA3 with gldA101-syn and the promoter, $\mathrm{P}_{\text {trc. }}$ & This study \\
\hline \multicolumn{3}{|l|}{ Strains } \\
\hline E. coli 10-Beta & Cloning host strain. & New England Biolabs \\
\hline $\begin{array}{l}\text { Synechocystis sp. } \\
\text { PCC } 6803\end{array}$ & Glucose tolerant wild type, naturally competent. & This study \\
\hline AV08 & 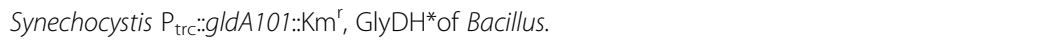 & This study \\
\hline AV10 & 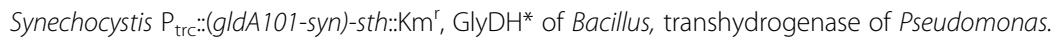 & This study \\
\hline AV11 & 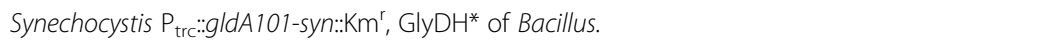 & This study \\
\hline
\end{tabular}


[32]. For segregation, the positive colonies were propagated continuously onto BG-11 plates containing kanamycin and segregation of colonies was verified through a colony PCR with the primers AMV17R and ps1_up_fwda (Table 1). The promoter and the heterologous genes in the engineered strains were PCR amplified with respective primers (ptka3-F, CO-F, O-F, sth-F) (Table 1) and sent for sequencing to Genewiz to verify the cloning accuracy.

\section{D (-) lactate analysis}

$\mathrm{D}(-) / \mathrm{L}(+)$ lactic acid detection kit (R-biopharm) was used to measure the D-lactate concentration. Samples of the cyanobacterial culture $(50 \mu \mathrm{L})$ were collected every 3 days and centrifuged at $12,000 \mathrm{rpm}$ for $5 \mathrm{~min}$. The supernatant was collected and the D-lactate concentration assay was performed following the manufacturer's instruction. All the reactions were performed in a 96well plate reader at room temperature (Infinite $200 \mathrm{PRO}$ microplate photometer, TECAN).

\section{${ }^{13} \mathrm{C}$ isotopomer experiment}

To estimate the carbon contributions of glucose and acetate for biomass and D-lactic acid synthesis a ${ }^{13} \mathrm{C}$ labeling experiment was performed. The mutant AV10 was grown in a BG-11 medium with $0.5 \%$ glucose $(1,2$ ${ }^{13} \mathrm{C}_{2}$ glucose) or $15 \mathrm{mM}$ acetate $\left(\mathrm{U}_{-}-{ }^{13} \mathrm{C}_{2}\right.$ acetate) (Sigma, St. Louis). Cultures were started at an $\mathrm{OD}_{730}$ of 0.4 and were grown with labeled glucose or acetate for over 48 hours. The biomass samples and supernatant were collected for measurement of lactate and amino acid labeling.

The proteinogenic amino acids from biomass were hydrolyzed and then derivatized with TBDMS [N-(tert-butyldimethylsilyl)- $N$-methyl-trifluoroacetamide], as described previously [39]. The derivatized amino acids were analyzed for their ${ }^{13} \mathrm{C}$ mass fraction by GC-MS (Hewlett Packard 7890A and 5975C, Agilent Technologies, USA) equipped with a DB5-MS column (J\&W Scientific) [39]. The fragment $[\mathrm{M}-57]^{+}$containing information of the entire amino acid was used for calculating the ${ }^{13} \mathrm{C}$ mass fractions (M: the molecular mass of the derivatized amino acids). The fragment $[\mathrm{M}-15]^{+}$was used only for leucine, since its $[\mathrm{M}-57]^{+}$ overlaps with other mass peak [40]. To analyze extracellular D-lactic acid labeling, the supernatant $(0.2 \mathrm{~mL})$ was first freeze-dried at $-50^{\circ} \mathrm{C}$. The dried samples were then prederivatized with $200 \mu \mathrm{L}$ of $2 \%$ methoxyamine hydrochloride in pyridine for 60 minutes at $37^{\circ} \mathrm{C}$ and then derivatized with $300 \mu \mathrm{L} N$-Methyl- $N$-(trimethylsilyl) trifluroacetamide (TMS) for 30 minutes at room temperature. The natural abundance of isotopes, including ${ }^{13} \mathrm{C}(1.13 \%),{ }^{18} \mathrm{O}(0.20 \%)$, ${ }^{29} \mathrm{Si}(4.70 \%)$ and ${ }^{30} \mathrm{Si}(3.09 \%)$ changes the mass isotopomer spectrum. These changes were corrected using a published algorithm and the detailed measurement protocol can be found in our previous paper [41].

\section{Additional file}

Additional file 1: Figure S1. Nuleotide sequence alignment of gldA101 and the codon-optimized gldA101 (i.e., gldA101-syn, synthesized by Genewiz Inc). Conserved nucleotide sequences in gldA101-syn are indicated as dotted lines. Figure S2. Autotrophic growth curve for Synechocystis 6803 strains shows similar growth of the engineered D-lactate producing strains as compared to the wild type strain. Diamond: Wild type. Square: AV08. Triangle: AV10. Circle: AV11. Figure S3. Mass spectra obtained via GC-MS confirm the presence of lactate in the cell culture supernatant of AV10 strain. D/L lactate enzyme kit (R-Biopharm) was used to further confirm that the product is an optically pure D-lactate.

\section{Competing interests}

The authors declare competing financial interests since this work is being covered by a pending patent application from Washington University in St. Louis.

\section{Authors' contributions}

AMV conceived the initial idea for this research. AMV, YY, and YJT designed the experiments. AMV, $Y Y$, and $Y L$ performed the experiments. All authors read and approved the manuscript.

\section{Acknowledgements}

We thank Professor K. T. Shanmugam for offering us the plasmid pQZ115. We thank Professor Himadri Pakrasi at WUSTL for his advice on this project. We also thank Dianyi Liu, Kanimozhi, and Zach Hembree for their help with experiments, and Sandra Matteucci from the WUSTL Engineering Communication Center, for her close reading of the manuscript. This research was funded by an NSF Career Grant (MCB0954016).

\section{Author details}

${ }^{1}$ Key Laboratory of Combinatory Biosynthesis and Drug Discovery (Ministry of Education), School of Pharmaceutical Sciences, Wuhan University, Wuhan 430071, China. ${ }^{2}$ Department of Energy, Environmental and Chemical Engineering, Washington University, St. Louis, MO 63130, USA.

Received: 26 September 2013 Accepted: 19 November 2013 Published: 25 November 2013

\section{References}

1. Frost JW, Draths KM, Knop DR, Harrup MK, Barker JL, Niu W: Chemicals from plants. In Carbon management: implications for $R \& D$ in the chemical sciences and technology (a workshop report to the chemical sciences roundtable). Washington, D.C: The National Academies Press; 2001.

2. Piringer OG, Baner AL: Plastic packaging: interactions with food and pharmaceuticals. 2nd edition. Weinheim: Wiley-VCH; 2008.

3. Vijayakumar J, Aravindan R, Viruthagiri T: Recent trends in the production, purification and application of lactic acid. Chem Biochem Eng Q 2008, 22:245-264.

4. Garlotta D: A literature review of poly (lactic acid). J Polym Environ 2001, 9:63-84.

5. Taskila S, Ojamo H: The current status and future expectations in industrial production of lactic acid by lactic acid bacteria. In Lactic acid bacteria - R \& D for food, health and livestock purposes. Edited by Kongo M. Rijeka, Croatia: InTech; 2013.

6. Atsumi S, Higashide W, Liao JC: Direct photosynthetic recycling of carbon dioxide to isobutyraldehyde. Nat Biotechnol 2009, 27:1177-1180.

7. Lan El, Liao JC: Metabolic engineering of cyanobacteria for 1-butanol production from carbon dioxide. Metab Eng 2011, 13:353-363.

8. Wang B, Pugh S, Nielsen DR, Zhang W, Meldrum DR: Engineering cyanobacteria for photosynthetic production of 3-hydroxybutyrate directly from $\mathrm{CO}_{2}$. Metab Eng 2013, 16:68-77.

9. Kusakabe T, Tatsuke T, Tsuruno K, Hirokawa Y, Atsumi S, Liao JC, Hanai T: Engineering a synthetic pathway in cyanobacteria for isopropanol production directly from carbon dioxide and light. Metab Eng 2013, 20C:101-108.

10. Wang B, Wang J, Zhang W, Meldrum DR: Application of synthetic biology in cyanobacteria and algae. Front Microbiol 2012, 3:344. 
11. Yu Y, You L, Liu D, Hollinshead W, Tang Y, Zhang F: Development of Synechocystis sp. PCC 6803 as a Phototrophic Cell Factory. Mar Drugs 2013, 11:2894-2916.

12. Berla BM, Saha R, Immethun CM, Maranas CD, Moon TS, Pakrasi HB: Synthetic biology of cyanobacteria: unique challenges and opportunities. Front Microbiol 2013, 4:246.

13. Wu GF, Shen ZY, Wu QY: Modification of carbon partitioning to enhance PHB production in Synechocystis sp PCC6803. Enzyme Microb Technol 2002, 30:710-715.

14. Xiao Y, Ruan ZH, Liu ZG, Wu SG, Varman AM, Liu Y, Tang YJJ: Engineering Escherichia coli to convert acetic acid to free fatty acids. Biochem Eng J 2013, 76:60-69.

15. Huang $\mathrm{H}-\mathrm{H}$, Camsund D, Lindblad P, Heidorn T: Design and characterization of molecular tools for a synthetic biology approach towards developing cyanobacterial biotechnology. Nucleic Acids Res 2010, 38:2577-2593

16. Huang $\mathrm{HH}$, Lindblad P: Wide-dynamic-range promoters engineered for cyanobacteria. J Biol Eng 2013, 7:10.

17. Joseph A, Aikawa S, Sasaki K, Tsuge Y, Matsuda F, Tanaka T, Kondo A: Utilization of lactic acid bacterial genes in Synechocystis sp. PCC 6803 in the production of lactic acid. Biosci Biotechnol Biochem 2013, 77:966-970.

18. Angermayr SA, Paszota M, Hellingwerf $\mathrm{KJ}$ : Engineering a cyanobacterial cell factory for production of lactic acid. Appl Environ Microbiol 2012, 78:7098-7106

19. Angermayr SA, Hellingwerf $\mathrm{KJ}$ : On the use of metabolic control analysis in the optimization of cyanobacterial biosolar cell factories. J Phys Chem $B$ 2013, 117:11169-11175.

20. Wang $Q$, Ingram LO, Shanmugam KT: Evolution of D-lactate dehydrogenase activity from glycerol dehydrogenase and its utility for D-lactate production from lignocellulose. Proc Natl Acad Sci 2011, 108:18920-18925.

21. McNeely K, Xu Y, Bennette N, Bryant DA, Dismukes GC: Redirecting reductant flux into hydrogen production via metabolic engineering of fermentative carbon metabolism in a cyanobacterium. Appl Environ Microbiol 2010, 76:5032-5038.

22. Niederholtmeyer $\mathrm{H}$, Wolfstadter BT, Savage DF, Silver PA, Way JC: Engineering cyanobacteria to synthesize and export hydrophilic products. Appl Environ Microbiol 2010, 76:3462-3466.

23. Axe DD, Bailey JE: Transport of lactate and acetate through the energized cytoplasmic membrane of Escherichia coli. Biotechnol Bioeng 1995, 47:8-19.

24. Campbell WH, Gowri G: Codon usage in higher-plants, green-algae, and cyanobacteria. Plant Physiol 1990, 92:1-11.

25. Lindberg P, Park S, Melis A: Engineering a platform for photosynthetic isoprene production in cyanobacteria, using Synechocystis as the model organism. Metab Eng 2010, 12:70-79.

26. Ungerer J, Tao L, Davis M, Ghirardi M, Maness P-C, Yu J: Sustained photosynthetic conversion of $\mathrm{CO}_{2}$ to ethylene in recombinant cyanobacterium Synechocystis 6803. Energy Environ Sci 2012, 5:8998-9006.

27. Vermaas WFJ: Photosynthesis and respiration in cyanobacteria. In eLS: John Wiley \& Sons, Ltd; 2001.

28. Tamoi M, Miyazaki T, Fukamizo T, Shigeoka S: The Calvin cycle in cyanobacteria is regulated by $C P 12$ via the $N A D(H) / N A D P(H)$ ratio under light/dark conditions. Plant J 2005, 42:504-513.

29. Takahashi $H$, Uchimiya $H$, Hihara $Y$ : Difference in metabolite levels between photoautotrophic and photomixotrophic cultures of Synechocystis sp. PCC 6803 examined by capillary electrophoresis electrospray ionization mass spectrometry. J Exp Bot 2008, 59:3009-3018.

30. Wermuth B, Kaplan NO: Pyridine nucleotide transhydrogenase from Pseudomonas aeruginosa: purification by affinity chromatography and physicochemical properties. Arch Biochem Biophys 1976, 176:136-143.

31. Bricker TM, Zhang S, Laborde SM, Mayer PR, Frankel LK, Moroney JV: The malic enzyme is required for optimal photoautotrophic growth of Synechocystis sp. Strain PCC 6803 under continuous light but Not under a diurnal light regimen. J Bacterio/ 2004, 186:8144-8148.

32. Varman AM, Xiao Y, Pakrasi HB, Tang YJ: Metabolic engineering of Synechocystis sp. Strain PCC 6803 for isobutanol production. Appl Environ Microbiol 2012, 79:908-914.

33. Wendisch VF, de Graaf AA, Sahm H, Eikmanns BJ: Quantitative determination of metabolic fluxes during coutilization of two carbon sources: comparative analyses with Corynebacterium glutamicum during growth on acetate and/or glucose. J Bacterio/ 2000, 182:3088-3096.
34. Heidorn $T$, Camsund D, Huang HH, Lindberg P, Oliveira P, Stensjo K, Lindblad P: Synthetic biology in cyanobacteria engineering and analyzing novel functions. Methods Enzymol 2011, 497:539-579.

35. Gao Z, Zhao H, Li Z, Tan X, Lu X: Photosynthetic production of ethanol from carbon dioxide in genetically engineered cyanobacteria. Energy Environ Sci 2012, 5:9857-9865.

36. Gründel M, Scheunemann R, Lockau W, Zilliges Y: Impaired glycogen synthesis causes metabolic overflow reactions and affects stress responses in the cyanobacterium Synechocystis sp. PCC 6803. Microbiology 2012, 158:3032-3043.

37. Brosius J, Erfle M, Storella J: Spacing of the -10 and -35 regions in the tac promoter. Effect on its in vivo activity. J Biol Chem 1985, 260:3539-3541.

38. Zang XN, Liu B, Liu SM, Arunakumara K, Zhang XC: Optimum conditions for transformation of Synechocystis sp. PCC 6803. J Microbiol 2007, 45:241-245.

39. You L, Page L, Feng X, Berla B, Pakrasi HB, Tang YJ: Metabolic pathway confirmation and discovery through ${ }^{13} \mathrm{C}$-labeling of proteinogenic amino acids. J Vis Exp 2012, 59:e3583.

40. Antoniewicz MR, Kelleher JK, Stephanopoulos G: Accurate assessment of amino acid mass isotopomer distributions for metabolic flux analysis. Anal Chem 2007, 79:7554-7559.

41. Tang Y, Shui W, Myers S, Feng X, Bertozzi C, Keasling J: Central metabolism in Mycobacterium smegmatis during the transition from $\mathrm{O}_{2}$-rich to $\mathrm{O}_{2}$-poor conditions as studied by isotopomer-assisted metabolite analysis. Biotechnol Lett 2009, 31:1233-1240.

\section{doi:10.1186/1475-2859-12-117}

Cite this article as: Varman et al:: Photoautotrophic production of D-lactic acid in an engineered cyanobacterium. Microbial Cell Factories 2013 12:117.

\section{Submit your next manuscript to BioMed Central and take full advantage of:}

- Convenient online submission

- Thorough peer review

- No space constraints or color figure charges

- Immediate publication on acceptance

- Inclusion in PubMed, CAS, Scopus and Google Scholar

- Research which is freely available for redistribution

Submit your manuscript at www.biomedcentral.com/submit
C) Biomed Central 